\title{
Ensemble disease gene prediction by clinical sample-based networks
}

\author{
Ping Luo ${ }^{1}$, Li-Ping Tian², Bolin Chen ${ }^{3}$, Qianghua Xiao ${ }^{4}$ and Fang-Xiang Wu $\mathbf{W}^{1,5,6,7^{*}}$ \\ From 6th International Work-Conference on Bioinformatics and Biomedical Engineering \\ Granada, Spain. 25-27 April 2018
}

\begin{abstract}
Background: Disease gene prediction is a critical and challenging task. Many computational methods have been developed to predict disease genes, which can reduce the money and time used in the experimental validation. Since proteins (products of genes) usually work together to achieve a specific function, biomolecular networks, such as the protein-protein interaction (PPI) network and gene co-expression networks, are widely used to predict disease genes by analyzing the relationships between known disease genes and other genes in the networks. However, existing methods commonly use a universal static PPI network, which ignore the fact that PPIs are dynamic, and PPIs in various patients should also be different.

Results: To address these issues, we develop an ensemble algorithm to predict disease genes from clinical sample-based networks (EdgCSN). The algorithm first constructs single sample-based networks for each case sample of the disease under study. Then, these single sample-based networks are merged to several fused networks based on the clustering results of the samples. After that, logistic models are trained with centrality features extracted from the fused networks, and an ensemble strategy is used to predict the finial probability of each gene being disease-associated. EdgCSN is evaluated on breast cancer (BC), thyroid cancer (TC) and Alzheimer's disease (AD) and obtains AUC values of 0.970, 0.971 and 0.966 , respectively, which are much better than the competing algorithms. Subsequent de novo validations also demonstrate the ability of EdgCSN in predicting new disease genes.

Conclusions: In this study, we propose EdgCSN, which is an ensemble learning algorithm for predicting disease genes with models trained by centrality features extracted from clinical sample-based networks. Results of the leave-one-out cross validation show that our EdgCSN performs much better than the competing algorithms in predicting $\mathrm{BC}$-associated, TC-associated and AD-associated genes. de novo validations also show that EdgCSN is valuable for identifying new disease genes.
\end{abstract}

Keywords: Disease gene prediction, Sample-based networks, Ensemble learning, Network centrality, Protein-protein interaction network

\section{Background}

Disease gene prediction is a critical yet challenging task. It helps us understand the mechanisms of diseases, find therapeutic targets, and develop novel treatment strategies [1]. During the past decades, disease gene prediction has gained great development. Many

\footnotetext{
*Correspondence: faw341@mail.usask.ca

${ }^{1}$ Division of Biomedical Engineering, University of Saskatchewan, S7N 5A9

Saskatoon, Canada

${ }^{5}$ Department of Computer Science, University of Saskatchewan, S7N 5C9

Saskatoon, Canada

Full list of author information is available at the end of the article
}

computational algorithms have been developed to predict disease genes so that the cost and time for in-depth validation could be maximumly reduced.

Among the various types of data that have been used to predict disease genes, protein-protein interactions (PPIs) are the most widely used evidence. On the one hand, interacting proteins (genes) usually have similar functions, which means algorithms can predict new disease genes based on their relationships with known disease genes in the PPI network. On the other hand, due to the network property of PPIs, most network analysis algorithms can

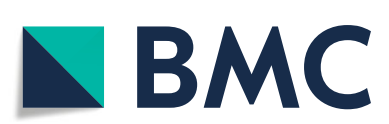

(c) The Author(s). 2020 Open Access This article is distributed under the terms of the Creative Commons Attribution 4.0 International License (http://creativecommons.org/licenses/by/4.0/), which permits unrestricted use, distribution, and reproduction in any medium, provided you give appropriate credit to the original author(s) and the source, provide a link to the Creative Commons license, and indicate if changes were made. The Creative Commons Public Domain Dedication waiver (http://creativecommons.org/publicdomain/zero/1.0/) applies to the data made available in this article, unless otherwise stated. 
be used to predict disease genes from PPI networks. For example, earlier methods, such as RWR, performed the random walk on PPI networks to predict disease genes [2]. Gillis et al. used degree centralities to rank all the genes [3].

However, PPIs are dynamic during the life time of cells, and not all PPIs exist in all the tissues. Static PPI networks downloaded from online databases contain lots of false positives which limit the performance of the methods that directly use them [4]. Thus, many studies integrate static PPI networks with disease-related data, such as GWAS and gene expression data, to improve the prediction accuracy [5-7]. This leads to two types of approaches. The first type of approaches weights PPI networks with disease-related data, and predicts candidate genes from the weighted networks. For instance, Wang et al. searched dense modules from a PPI network weighted by gene expression and GWAS data [6]. Our previous study trained a regression model with features extracted from a PPI network weighted by differential co-expression [8]. The second type of approaches constructs heterogeneous networks and combines them with PPI networks to enhance the prediction. For example, Chen et al. combined gene co-expression networks and pathway coexist networks with PPI networks to predict disease genes [9, 10]. Singh-Blom et al. trained a biased SVM with features extracted from phenotype-phenotype networks and PPI networks [11] to predict disease genes. Despite their success, the discussed approaches still use PPI networks with false positive interactions, which contain inaccurate topological structures. PPI networks downloaded from different databases might affect the prediction results.

To solve these issues, in our previous study, gene expression data of clinical samples have been used to construct sample-specific PPI networks [12]. Each single samplebased network only contains the significant PPIs associated with the disease under consideration, which reduces the false positive interactions. A network that fuses all the single sample-based networks was used to predict the disease-associated genes, so that disease genes that function in different patients could all be identified. In this study, to further extend our research, an ensemble algorithm that predicts disease genes from clinical samplebased networks (EdgCSN) is proposed. Meanwhile, Katz centrality is used instead of edge clustering coefficient to better extract local structural information from the sample-based networks.

\section{Methods}

Figure 1 depicts the work flow of EdgCSN which is explained as follows. (a)-(b). A single sample-based network is constructed for each case sample by combining clinical samples and the universal static PPI network. (c). The case samples are clustered into a few groups and single sample-based networks of the samples in the same group are fused to one network. (d). A logistic model is trained by the centrality features extracted from each fused network, and the probability of each gene being disease-associated is predicted. (e). The maximum probability of a gene calculated from all the logistic models is regarded as its probability of being disease-associated. In the following subsections, details of the five steps in Fig. 1 are first discussed. Then, the data sources and evaluation metrics are explained.

\section{Sample-based networks}

To obtain the most informative PPIs and remove the false positive ones, sample-based networks are used in this study instead of the universal static PPI networks. In addition, since the real caustic genes of different patients may not be the same, case samples are divided into different clusters so that patients with distinct conditions are analyzed separately. Specifically, three steps are performed to obtain the sample-based networks.

1 A single sample-based network is constructed for each case sample;

2 Case samples are classified into different clusters;

3 Networks of the samples in the same cluster are fused together.

For the first step, we assume that a PPI exists in a single sample-based network $N_{s}$ only if the two interacted proteins are both activated in sample $s$. Concretely, a gene $i$ in a case sample $s$ is considered being activated if

$$
\operatorname{mcase}[i, s] \geq \lambda * \operatorname{mean}(\operatorname{mcntl}[i])
$$

where mcase $[i, s]$ is the expression value of gene $i$ in sample $s$, and mean $(\operatorname{mcnt}[i])$ is the mean expression value of gene $i$ over all control samples. To construct $N_{s}$, every edge $(i, j)$ in the static PPI network is validated and only the one with both $i$ and $j$ being activated is added to $N_{s}$. Then, $S$ single sample-based networks are constructed for the $S$ case samples.

For the second step, hierarchical clustering is used to classify case samples into different clusters. Given two samples $s_{1}$ and $s_{2}$, their pairwise distance is calculated by

$$
\operatorname{dist}\left(s_{1}, s_{2}\right)=1-\frac{\left(\mathbf{s}_{1}-\overline{\mathbf{s}}_{1}\right) \cdot\left(\mathbf{s}_{2}-\overline{\mathbf{s}}_{2}\right)}{\left\|\mathbf{s}_{1}-\overline{\mathbf{s}}_{1}\right\|_{2}\left\|\mathbf{s}_{2}-\overline{\mathbf{s}}_{2}\right\|_{2}}
$$

where $\mathbf{s}_{1}\left(\mathbf{s}_{2}\right)$ is a vector of expression values of genes in sample $s_{1}\left(s_{2}\right)$, and $\overline{\mathbf{s}}_{1}\left(\overline{\mathbf{s}}_{2}\right)$ is the corresponding average expression value. During the bottom-up process, distance between two newly formed clusters $u$ and $v$ is computed as follows

$$
\operatorname{Distance}(u, v)=\max _{p \in u, q \in v}(\operatorname{dist}(p, q))
$$




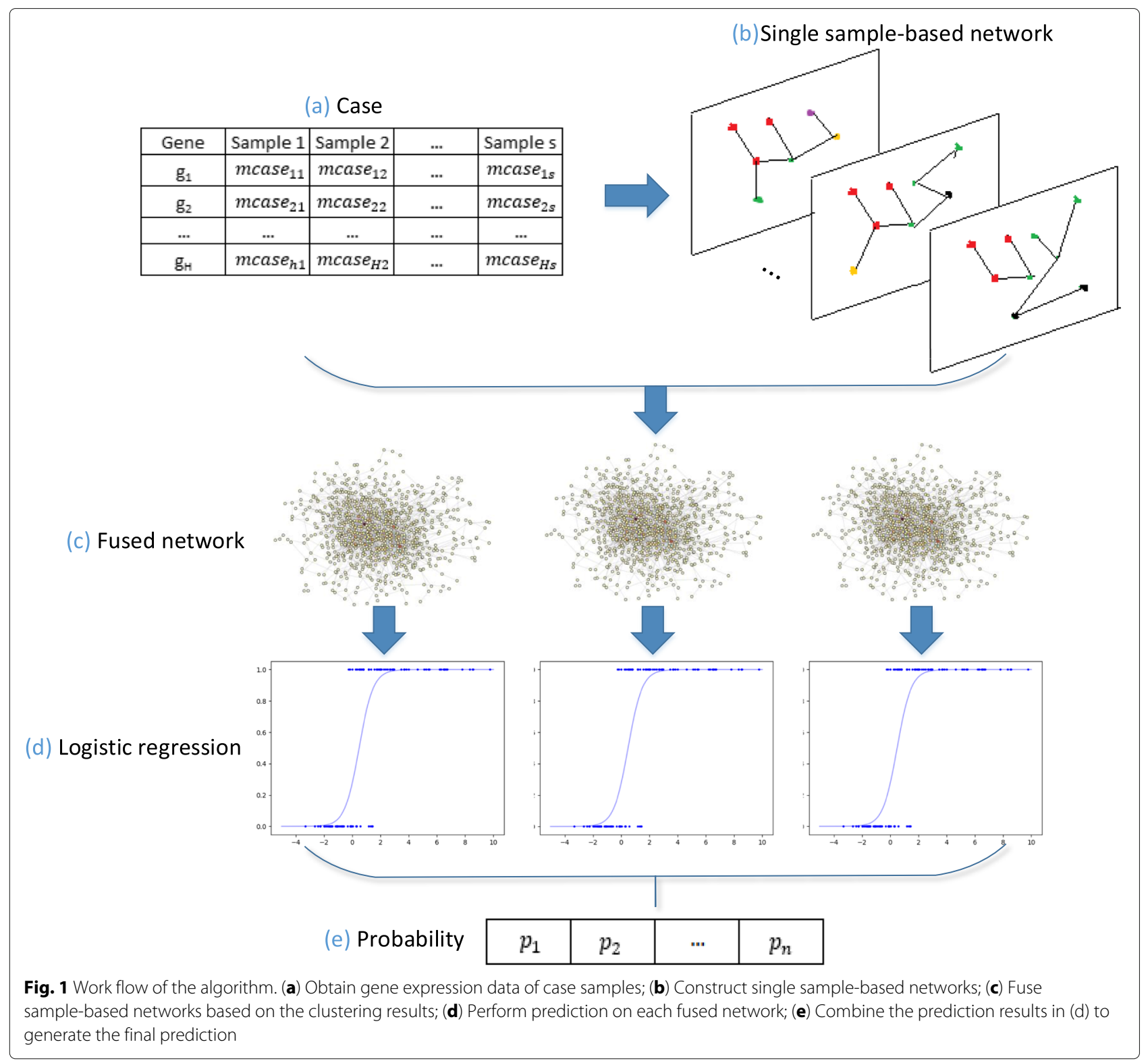

which is the maximum distance between samples in $u$ and $v$. Let dmax denote the maximum distance among clusters, $0.7 * d$ max is used as the threshold to select clusters from the resulted dendrogram.

For the third step, assuming all the $S$ samples are classified into $l$ clusters and the $t$-th cluster contains $S_{t}$ samples, we have $S=\sum_{t=1}^{l} S_{t}$. The objective is to fuse the networks of the samples in the same cluster into one network. Although many network fusion methods have been published [13], most of them cannot efficiently fuse complex PPI networks, especially when the number of networks to be fused is more than 1,000 . Thus, we propose a simple strategy which uses a threshold $\epsilon$ to determine whether an edge exists in the fused networks. An edge $(i, j)$ is considered as significant only if it appears in at least $\epsilon$ single sample-based networks. Precisely, given a cluster with $S_{t}$ samples, let $f_{i j}$ be the number of times edge $(i, j)$ appears in the $S_{t}$ single sample-based networks. When $f_{i j}<\epsilon,(i, j)$ is not included in the fused network, and when $f_{i j} \geq \epsilon$, $(i, j)$ is in the fused network. Finally, $l$ fused networks are obtained for the $l$ clusters, respectively.

\section{Model design}

Given a biomolecular network, if disease genes are labeled as 1 and non-disease genes are labeled as 0 , the disease gene prediction problem can then be formulated as a network labeling problem [14]. Let $\mathbf{x}=\left(x_{1}, x_{2}, \ldots, x_{H}\right)$ denote a set of binary labels of all the $H$ genes in the biomolecular network. $\mathbf{x}$ is known as the configuration of the network, and the set $X$ of all possible configurations is 
a random field. Based on our previous studies [8, 10, 15], a generalized model was proposed in [12] which predicted the probability of a gene $i$ being labeled as 1 by

$$
P\left(x_{i}=1 \mid x_{[-i]}, \theta\right)=\frac{\exp \left(\theta \phi_{i}\right)}{1+\exp \left(\theta \phi_{i}\right)}
$$

where $\theta$ is a parameter vector and $\phi_{i}$ is the feature vector of gene $i$ extracted from the biomolecular network labeled by a prior configuration $\mathbf{x}$.

In [12], $\phi_{i}$ is a 7-dimensional feature vector which consists of a dummy feature (1) and three pairs of $0-1$ centrality features: $0-1$ degree centrality, $0-1$ closeness centrality and $0-1$ edge clustering coefficient. These three $0-1$ centrality indices have shown their ability in characterizing discriminative features for classifying disease and non-disease genes. However, edge clustering coefficient can only capture the structural information between genes and their direct neighbors, and the relations between genes and their $\mathrm{k}$-th order $(k \geq 2)$ neighbors cannot be obtained. Since proteins usually form a complex or functional module to achieve a specific function [4], the k-th order neighbors should also be considered when the local structural information is extracted. Previous study also showed that the indirect neighbors were useful for disease gene prediction [16]. Thus, we replace edge clustering coefficient by Katz centrality in this study to leverage the local structure information between nodes and their higher order neighbors.

Given a labeled network $N=(V, E), V$ is the set of nodes and $E$ is the set of edges, the $0-1$ degree centrality denoted by $C_{i 0}^{d}$ and $C_{i 1}^{d}$ are defined as follows

$$
C_{i 0}^{d}=\sum_{(i, j) \in E}\left(1-x_{j}\right), \quad C_{i 1}^{d}=\sum_{(i, j) \in E} x_{j}
$$

The $0-1$ closeness centrality denoted by $C_{i 0}^{c}$ and $C_{i 1}^{c}$ are defined as

$$
\begin{aligned}
C_{i 0}^{c} & =\frac{1}{n_{0}-1} \sum_{j \in V, j \neq i} \frac{1}{d s p(i, j)}\left(1-x_{j}\right), \\
C_{i 1}^{c} & =\frac{1}{n_{1}-1} \sum_{j \in V, j \neq i} \frac{1}{d s p(i, j)} x_{j}
\end{aligned}
$$

where $d s p(i, j)$ is the length of the shortest path between node $i$ and $j, n_{0}$ and $n_{1}$ are the number of nodes labeled as 0 and 1 , respectively

Katz centrality measures the relative influence of a node in the network [17]. It is defined by

$$
C_{i}^{k}=\sum_{k=0}^{\infty} \sum_{j=1}^{n} \alpha^{k}\left(A^{k}\right)_{j i}
$$

where $A$ is the adjacency matrix of the network, $k$ is the length of the path between $i$ and $j, \alpha$ is a damping factor penalizes the impact node $j$ on $i$. The longer the path, the smaller the impact node $j$ is on $i$.
When $\alpha$ is properly chosen, Eq. (7) will converge as $k \rightarrow$ $\infty$. However, when Katz centrality is used in this study, we care more about the information conveyed by paths with short distance (less than 5). Study in link prediction also showed that $k=3$ or $k=4$ can yield satisfactory performance [18]. Thus, $\alpha$ and $k$ are chosen by grid search without the proof of convergence.

In previous studies, Katz centrality calculated from heterogeneous networks had been used to prioritize disease genes [11]. However, results of directly using Katz centrality were not better than existing methods, such as RWR [2]. To make Katz centrality suitable for disease gene prediction, we define the $0-1$ Katz centrality as follows:

$$
\begin{aligned}
C_{i 0}^{k} & =\sum_{k=0}^{\infty} \sum_{j=1}^{n} \alpha^{k}\left(A^{k}\right)_{j i}\left(1-x_{j}\right), \\
C_{i 1}^{k} & =\sum_{k=0}^{\infty} \sum_{j=1}^{n} \alpha^{k}\left(A^{k}\right)_{j i} x_{j}
\end{aligned}
$$

Similar to $0-1$ degree and $0-1$ closeness centrality, the $0-1$ Katz centrality measures the importance of a gene among disease genes and non-disease genes, respectively, which is more appropriate for disease gene prediction. The new feature vector of each gene is then defined as

$$
\phi_{i}=\left(1, C_{i 0}^{d}, C_{i 1}^{d}, C_{i 0}^{c}, C_{i 1}^{c}, C_{i 0}^{k}, C_{i 1}^{k}\right)
$$

\section{Network labeling and benchmark selection}

As discussed in the previous section, biomolecular networks are needed to be labeled by a prior configuration so that disease genes can be predicted. In this study, we use the $l$ fused networks to predict disease genes, which means the known disease genes in these networks are labeled as 1 while other genes are labeled as 0 . Then, the feature vectors of all genes can be extracted by Eq. (9).

In addition, to train the logistic models used for prediction, we also need a set of non-disease genes, which are used as negative instances. Unfortunately, no databases contain non-disease genes. Therefore, our previous strategy proposed in [19] is used to select the non-disease genes used in the training.

In [19], a disease gene network (DGN) was constructed with the disease-gene association data downloaded from OMIM [20]. In the DGN, each node is either a disease or a disease-associated gene. Diseases are connected with their associated genes, and two diseases are connected if they share one or more associated genes. Thus, diseases that are close to each other in the DGN have more chances to share similar disease genes, which means they are more likely to have similar mechanisms. If the length of the shortest path between two diseases is larger than a threshold $\eta$, they might not have similar mechanisms, and the disease genes of one disease could be regarded as 
non-disease genes of the other disease. With this strategy, a group of non-disease genes are obtained for the disease under study, and only non-disease genes that exist in all the $l$ fused networks are selected. $\eta=5$ is chosen based on our previous experience.

Assuming $m$ disease genes are known to be associated with the disease under study, we randomly select $m$ genes from the set of non-disease genes, and these $2 m$ genes form a set of gold standard genes. This process is performed 50 times and finally we obtain 50 sets of gold standard genes and regarded them as benchmarks.

\section{Ensemble prediction}

Given $m$ disease genes and $m$ non-disease genes, features of these genes extracted from the $l$ fused networks are used to train $l$ logistic models, respectively. Equation (4) is then used to predict the probability of each gene being disease-associated in each fused network.

For each gene, $l^{\prime}\left(1 \leq l^{\prime} \leq l\right)$ probabilities are calculated. Considering that the caustic genes of different samples might be different, the obtained probabilities only reveal the potential of the gene being disease-associated in the corresponding clusters. Thus, for each gene, the ensemble strategy chooses the maximum value of the $l^{\prime}$ probabilities as its probability of being disease-associated.

\section{Datasets}

In this study, datasets of breast cancer (BC), thyroid cancer (TC) and Alzheimer's disease (AD) are used to evaluate the algorithm. The $\mathrm{BC}$-associated genes and TCassociated genes are obtained from the Cancer Gene Census category (http://cancer.sanger.ac.uk/census) [21]. In total, $35 \mathrm{BC}$-associated genes and $33 \mathrm{TC}$-associated genes are used as the benchmarks. The $\mathrm{AD}$-associated genes are obtained from MalaCards: The human disease database (http://www.malacards.org/). The database contains 182 potential $\mathrm{AD}$ associated genes ranked by their probability of being $\mathrm{AD}$-associated in descending order. 39 of the first 50 genes exist in the static PPI network are used as benchmarks.

The gene expression data of $\mathrm{BC}$ and $\mathrm{TC}$ are downloaded from NCI Genomic Data Commons (GDC) [22], which measures the data by RNA-Seq. We download the data normalized by FPKM (Fragments Per Kilobase Million) and transform them to TPM (Transcripts Per Kilobase Million) by the strategy proposed in [23]. The expression data of Alzheimer's disease (AD) are downloaded from Gene Expression Omnibus (GSE53697) [24], which are also measured by RNA-seq. The data normalized by RPKM (Reads Per Kilobase Million) are downloaded and transformed to TPM with the same strategy used for the data downloaded from GDC. TPM is chosen because it facilitates the comparison of the proportion of reads that are mapped to a gene in each sample and is usually better than FPKM and RPKM in cross-sample comparison, which helps us properly cluster all the samples. In total, the dataset of $\mathrm{BC}$ contains 1102 case samples and 113 control samples; the dataset of TC contains 502 case samples and 58 control samples; the dataset of $\mathrm{AD}$ contains 9 case samples and 8 control samples.

After downloading the gene expression data, four steps are performed to control the genes used in our study. (1). TPM values less than 1 are replaced by 0 because of the unreliability. (2). $\log _{2}(\mathrm{TPM}+1)$ is used instead of the original TPM values. (3). Genes expressed in less than $10 \%$ of samples (case and control) are removed. (4). Genes not existing in the PPI network are removed. In total, 14436 genes, 13959 genes and 13370 genes are left for BC dataset, TC dataset and AD dataset, respectively.

The static PPI network is downloaded from the InWeb_InB-ioMap database (version 2016_09_12) [25]. The database consists of more than 600,000 protein interactions collected from eight source databases, which insures that valuable protein interactions are not missed during the construction of the sample-based PPI networks. In this study, the proteins in the PPI network are mapped to their corresponding genes to form a gene-gene interaction network. In the paper, the term "PPI network" is used to represent the gene-gene interaction network because of simplicity.

\section{Evaluation metrics}

In this study, a disease gene is regarded as positive while a non-disease gene is regarded as negative. Given a threshold $\Gamma$, a gene $i$ with a probability $p_{i} \geq \Gamma$ is predicted as positive, and otherwise it is predicted as negative. For all genes in the benchmark, the true positives (TP), false positives (FP), true negatives (TN), and false negatives (FN) are defined as follows

1 TP: a disease gene is predicted as a disease gene

2 FP: a non-disease gene is predicted as a disease gene

$3 T N$ : a non-disease gene is predicted as a non-disease gene

$4 F N$ : a disease gene is predicted as a non-disease gene

Then, we can calculate the true positive rate (TPR) and the false positive rate (FPR) of the prediction results by the following equations

$$
T P R=\frac{T P}{T P+F N}, F P R=\frac{F P}{T N+F P}
$$

To evaluate the algorithm, the receiver operating characteristic (ROC) curve is created by plotting the TPR against FPR with various $\Gamma$. The area under the ROC curve (AUC) is also used to evaluate the overall performance of the algorithm.

Since the number of genes used as benchmark is small, leave-one-out cross validation (LOOCV) is performed 


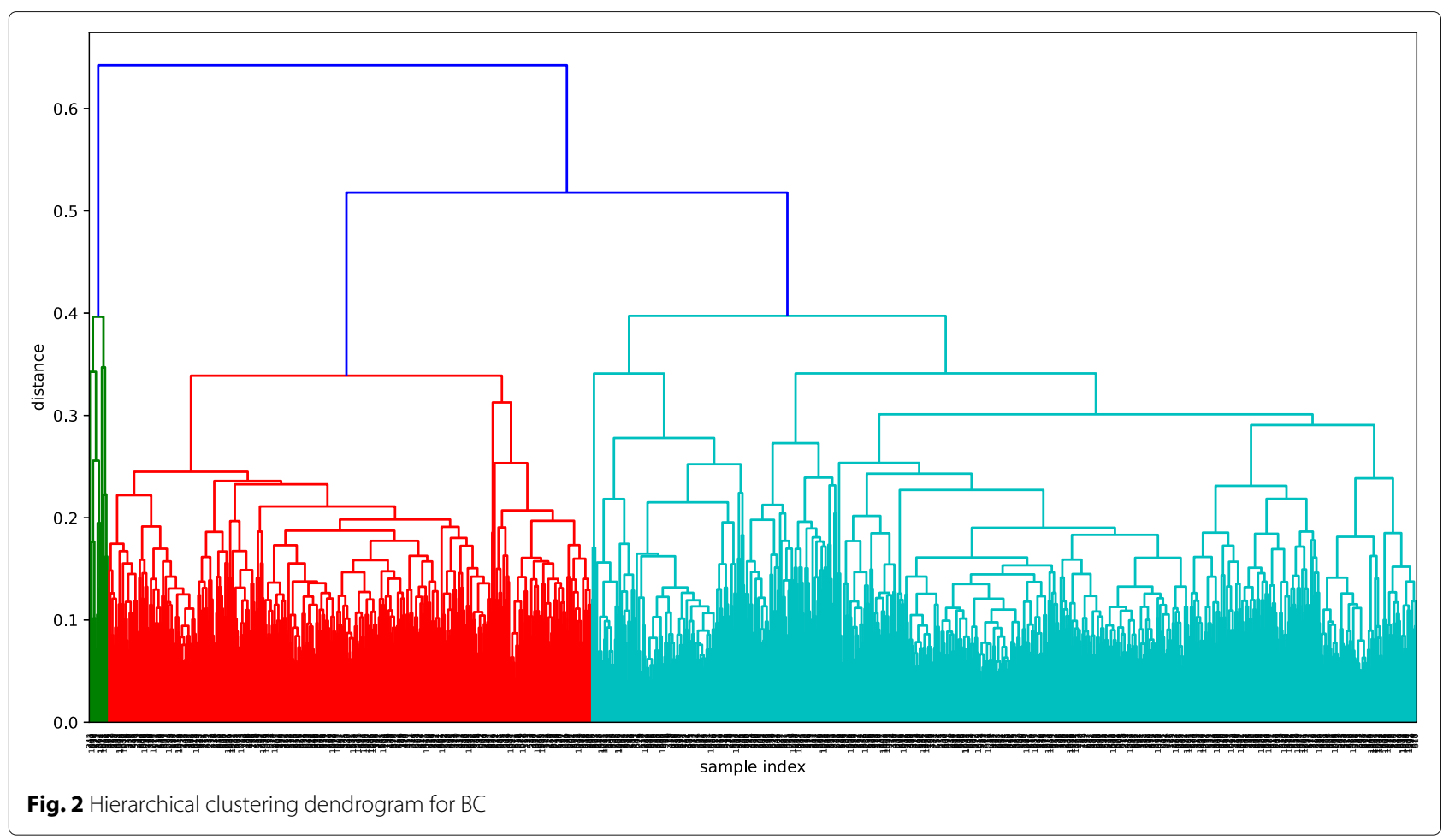

to calculate the probabilities of genes in the benchmark being disease-associated. With the 50 sets of gold standard genes, LOOCV is performed 50 times. In each round, the probabilities of the $2 m$ genes being diseaseassociated are calculated, as well as the AUC value.
The average AUC value is then used to evaluate the algorithm.

In addition, de novo validation is performed by ranking all the unknown genes in descending order by their average probabilities calculated by the models trained

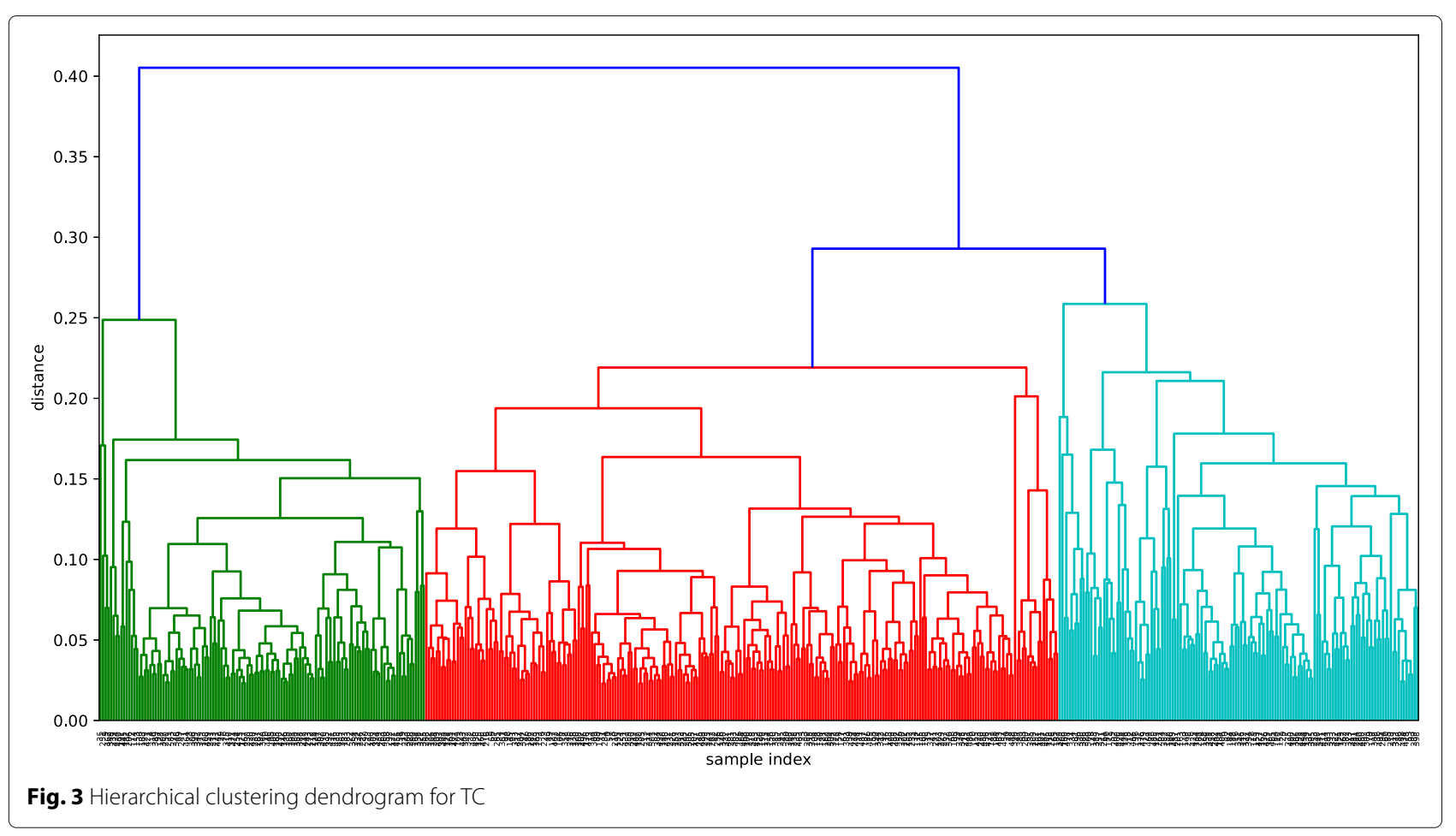




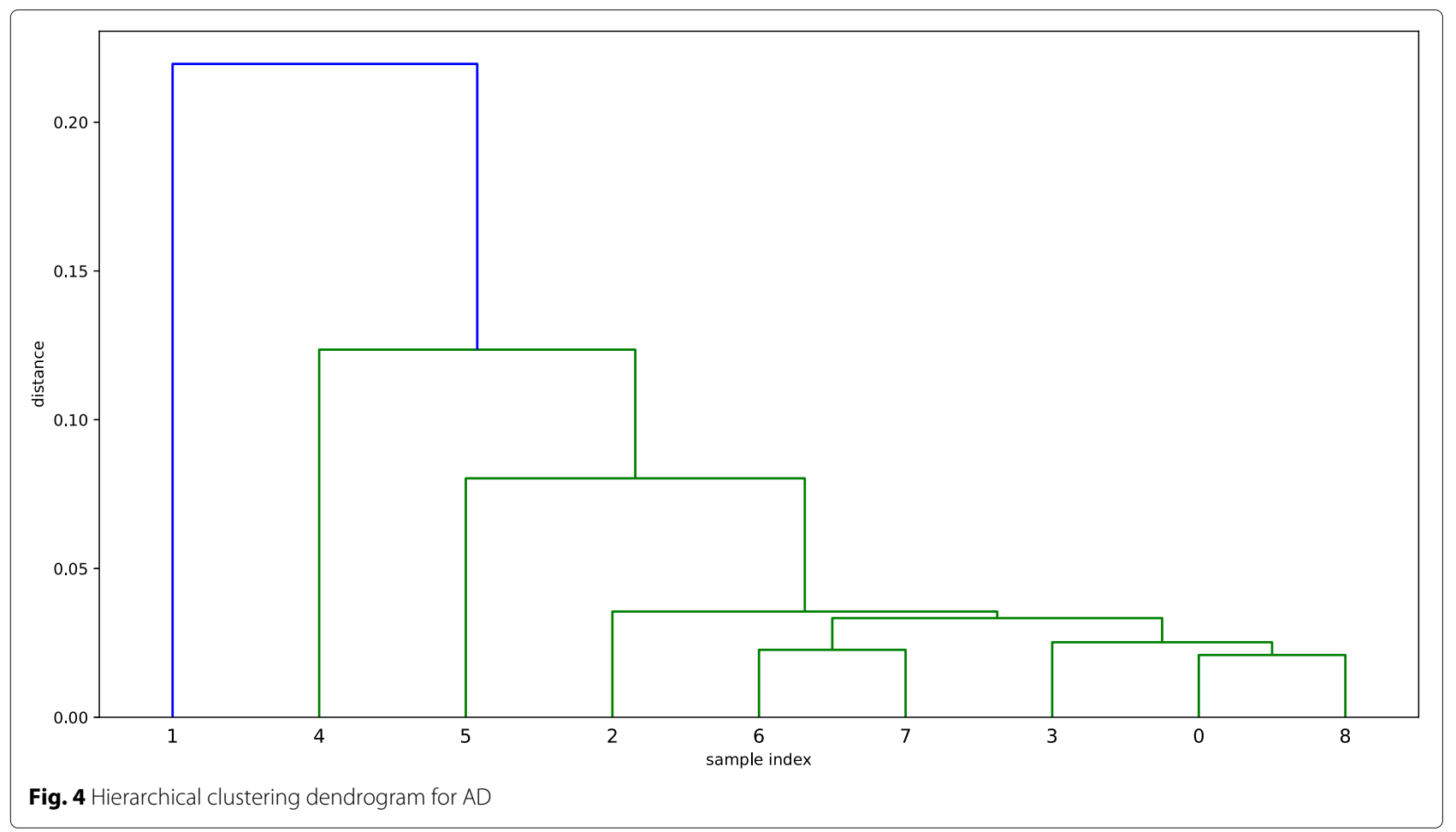

with the 50 sets of gold standard genes. The top 10 unknown genes are analyzed from published literature to illustrate the ability of EdgCSN in predicting new disease genes.

\section{Results}

\section{Clustering}

Figures 2, 3 and 4 show the dendrograms of the hierarchical clustering. $\mathrm{BC}$ and $\mathrm{TC}$ samples are divided to three clusters and $\mathrm{AD}$ samples are divided to two clusters. Thus, three fused networks are constructed for BC and

Table 1 Sensitivity analysis

\begin{tabular}{|c|c|c|c|c|c|}
\hline \multirow[b]{2}{*}{$\lambda$} & \multirow[b]{2}{*}{$\alpha$} & \multicolumn{4}{|l|}{ k } \\
\hline & & 1 & 2 & 3 & 4 \\
\hline 1.0 & 0.1 & 0.867 & 0.961 & 0.873 & 0.878 \\
\hline 1.0 & 0.2 & 0.869 & 0.966 & 0.889 & 0.870 \\
\hline 1.1 & 0.1 & 0.883 & 0.967 & 0.890 & 0.903 \\
\hline 1.1 & 0.2 & 0.881 & 0.970 & 0.909 & 0.896 \\
\hline 1.2 & 0.1 & 0.845 & 0.957 & 0.877 & 0.898 \\
\hline 1.2 & 0.2 & 0.846 & 0.958 & 0.892 & 0.894 \\
\hline 1.3 & 0.1 & 0.787 & 0.938 & 0.819 & 0.842 \\
\hline 1.3 & 0.2 & 0.787 & 0.940 & 0.841 & 0.842 \\
\hline 1.5 & 0.1 & 0.777 & 0.938 & 0.813 & 0.775 \\
\hline 1.5 & 0.2 & 0.777 & 0.938 & 0.786 & 0.816 \\
\hline
\end{tabular}

TC, respectively, and two fused networks are constructed for $\mathrm{AD}$.

\section{Sensitivity analysis}

The performance of our algorithm is affected by four hyperparameters: $\lambda, \epsilon, \alpha$ and $k$. The first two control the resulted fused networks. Based on our previous study, edges that exist in more than three networks were significant [12]. Thus, $\epsilon=3$ is empirically chosen in this study. As for $\lambda$, since the RNA-seq data are normalized by TPM rather than DESeq2 [26], $\lambda$ is searched from a

Table 2 Sensitivity analysis

\begin{tabular}{llllll}
\hline & & $k$ & & & \\
\cline { 3 - 6 }$\lambda$ & $\alpha$ & 1 & 2 & 3 & 4 \\
\hline 1.0 & 0.1 & 0.716 & 0.966 & 0.839 & 0.790 \\
1.0 & 0.2 & 0.713 & 0.967 & 0.795 & 0.802 \\
1.1 & 0.1 & 0.729 & $\mathbf{0 . 9 7 1}$ & 0.800 & 0.746 \\
1.1 & 0.2 & 0.728 & 0.969 & 0.744 & 0.779 \\
1.2 & 0.1 & 0.809 & 0.954 & 0.748 & 0.776 \\
1.2 & 0.2 & 0.808 & 0.953 & 0.652 & 0.792 \\
1.3 & 0.1 & 0.621 & 0.962 & 0.779 & 0.786 \\
1.3 & 0.2 & 0.620 & 0.960 & 0.662 & 0.794 \\
1.5 & 0.1 & 0.412 & 0.965 & 0.809 & 0.720 \\
1.5 & 0.2 & 0.411 & 0.963 & 0.645 & 0.679 \\
\hline
\end{tabular}

The resulted AUC values obtained with different combinations of hyperparameters for TC

The highest AUC value is marked in boldface 
Table 3 Sensitivity analysis

\begin{tabular}{llllll}
\hline & & & & & \\
\cline { 3 - 6 }$\lambda$ & $\alpha$ & 1 & 2 & 3 & 4 \\
\hline 1.0 & 0.1 & 0.808 & 0.964 & 0.809 & 0.763 \\
1.0 & 0.2 & 0.809 & $\mathbf{0 . 9 6 6}$ & 0.764 & 0.705 \\
1.1 & 0.1 & 0.665 & 0.956 & 0.757 & 0.685 \\
1.1 & 0.2 & 0.665 & 0.957 & 0.596 & 0.636 \\
1.2 & 0.1 & 0.564 & 0.938 & 0.809 & 0.605 \\
1.2 & 0.2 & 0.563 & 0.939 & 0.608 & 0.596 \\
1.3 & 0.1 & 0.508 & 0.914 & 0.810 & 0.674 \\
1.3 & 0.2 & 0.508 & 0.914 & 0.608 & 0.614 \\
\hline
\end{tabular}

The resulted AUC values obtained with different combinations of hyperparameters for $A D$

The highest AUC value is marked in boldface

new set $\{1.0,1.1,1.2,1.3,1.5\}$, which is different from the one obtained in our previous study. The other two hyperparameters control the information extracted by Katz centrality. To obtain the appropriate hyperparameters, $\alpha$ is searched from $\{0.1,0.2\}$, and $k$ is searched from $\{1,2,3,4\}$, respectively.

Tables 1, 2 and 3 show the results of the grid search for $\mathrm{BC}, \mathrm{TC}$ and $\mathrm{AD}$, respectively. EdgCSN performs best for $\mathrm{BC}$ when $\lambda=1.1, \alpha=0.2, k=2$ with an AUC $=$ 0.970; for TC when $\lambda=1.11, \alpha=0.1, k=2$ with an $\mathrm{AUC}=0.971$; for $\mathrm{AD}$ when $\lambda=1.0, \alpha=0.2, k=2$ with an AUC $=0.966$. ' - ' denotes that more than $10 \%$ known disease genes are not contained in the fused networks constructed by the corresponding hyperparameters.

All the three experiments obtain their best AUC values when $k=2$, and a smaller or higher $k$ would significantly affect the performance of the algorithm. These results indicate that local structural information contained within the second order neighborhood is valuable for disease gene prediction. Other disease gene prediction algorithms that use topological structure of biomolecular networks could also further include these information to improve their prediction.

\section{Comparison}

EdgCSN is compared with three algorithms: the Rebalanced algorithm of Chen et al. [10], the AIDG algorithm of Tang et al. [27], and our previous algorithm dgCSN [12]. Re-balanced method combined multiple types of biomolecular networks to predict cancer-related genes, and AIDG used sub-cellular localization to purify universal PPI networks. These algorithms have been shown better than many classical methods, such as the RWR method [2], the DIR method [28] and the ToppNet [29].

The resulted $\mathrm{ROC}$ curves for $\mathrm{BC}, \mathrm{TC}$, and $\mathrm{AD}$ are depicted in Figs. 5, 6, 7, respectively. The AUC values of EdgCSN for BC, TC and AD are 0.970, 0.971 and 0.966, respectively, which are much better than those of the competing algorithms. For BC, our EdgCSN is 7\% more accurate than the competing algorithms, and for $\mathrm{TC}$ and $\mathrm{AD}, \mathrm{EdgCSN}$ is $20 \%$ more accurate than the other three algorithms.

\section{de novo validation}

To validate the performance of EdgCSN in predicting new disease genes, unknown genes are ranked in descending order by their average probabilities of being

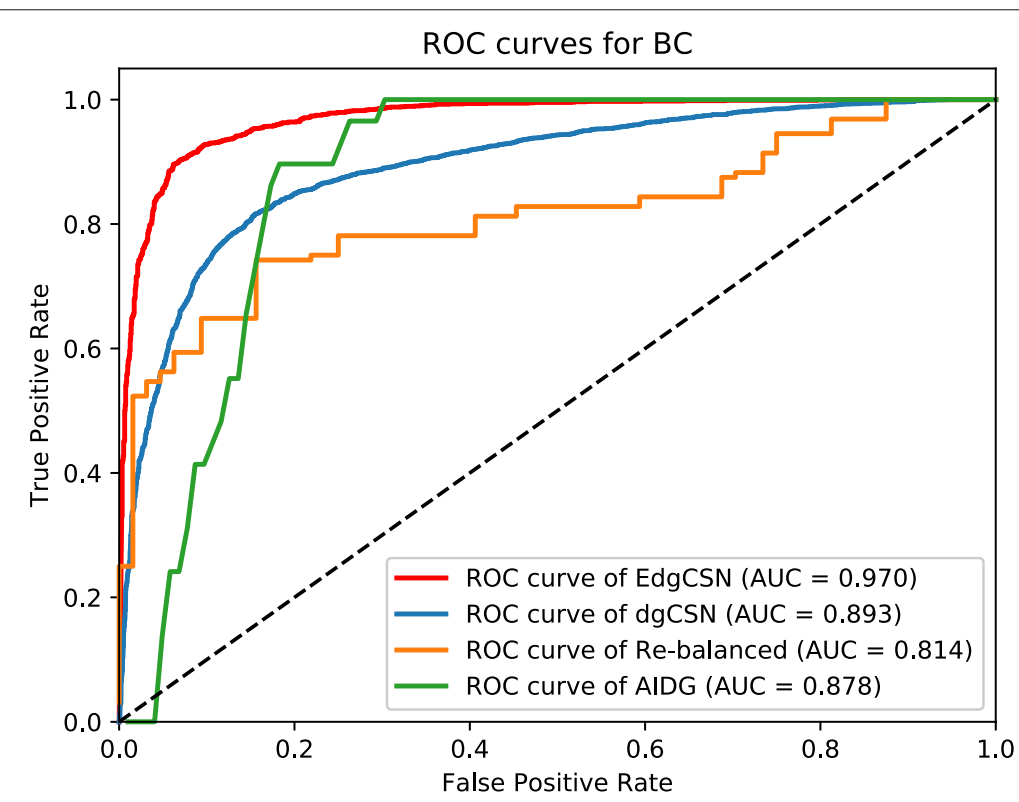

Fig. 5 ROC curves for $B C$ 


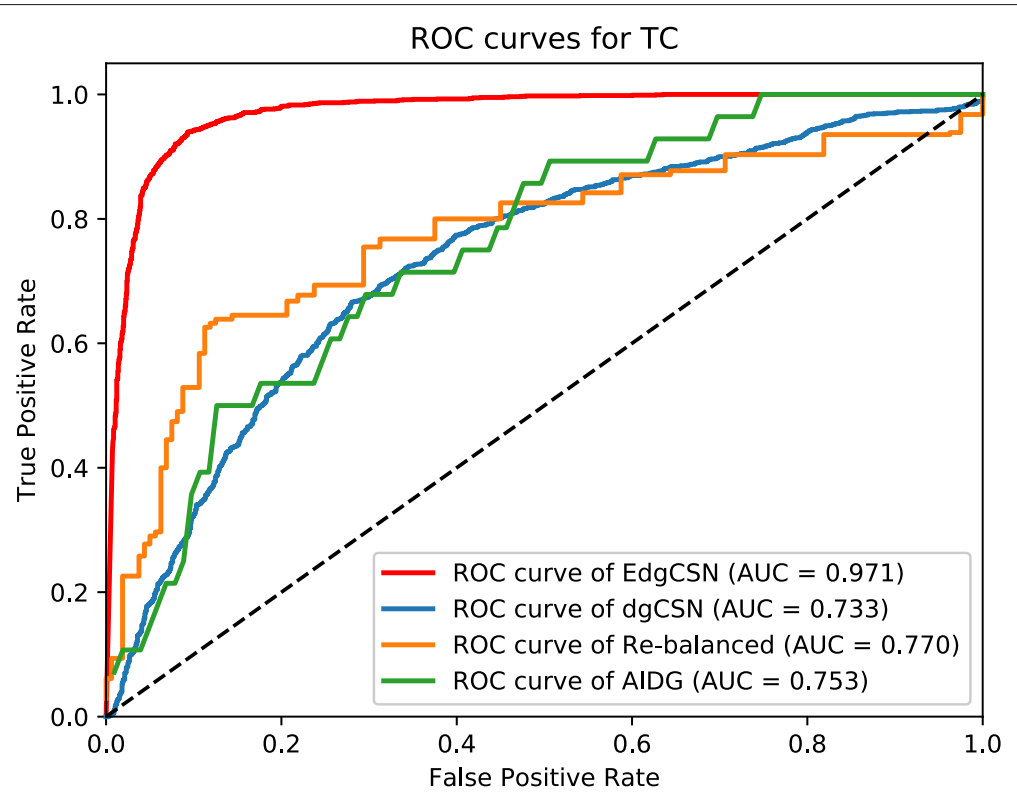

Fig. 6 ROC curves for TC

disease-associated predicted by the 50 sets of genes in the benchmark. The top 10 predictions are further searched in existing literature to find out if they are associated with the disease under study.

Table 4 shows the top 10 predictions of the three diseases. Functions of the genes that have not been studied in existing literature are left blank. Most of the genes have been analyzed as disease-associated in existing studies, especially for $\mathrm{BC}$, where all the 10 genes have been studied in the existing literature. For TC, although only 5 of the 10 genes have been studied, 3 of the 5 genes that have not been studied ('CEP72', 'CEP131' and 'GPR83') belong to the Centrosomal Protein family and G Proteincoupled Receptor respectively. Many proteins belong to these families are closely related to cancers [30], which means 'CEP72', 'CEP131' and 'GPR83' might be predicted as being $\mathrm{TC}$-associated in the future.

\section{Discussion}

Many algorithms have been proposed to predict disease genes, and most of them rely on PPI networks to achieve the prediction. However, PPI is dynamic and tissue-

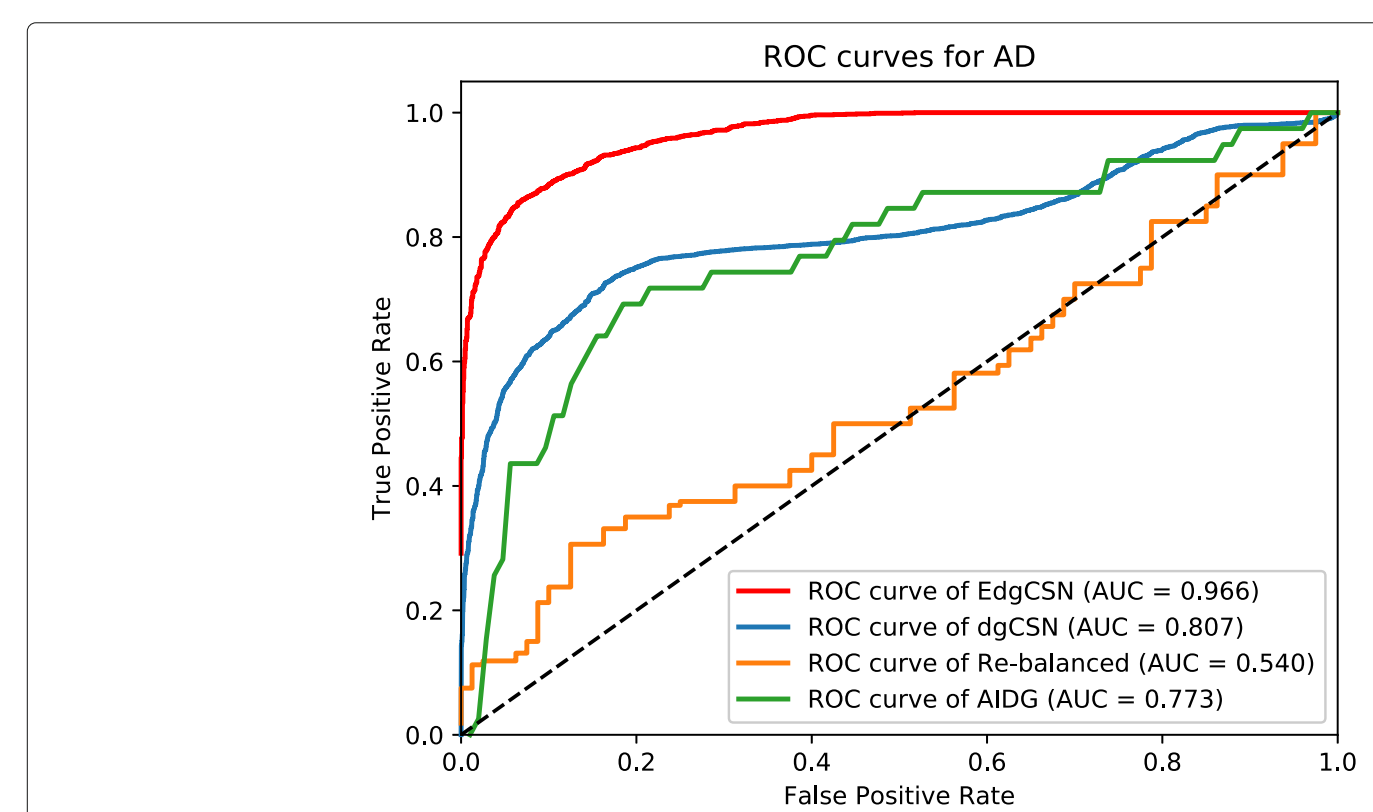

Fig. 7 ROC curves for $A D$ 
Table 4 Top 10 unknown genes

\begin{tabular}{|c|c|c|}
\hline Gene Name & Function & Reference \\
\hline \multicolumn{3}{|l|}{ BC } \\
\hline CREBBP & Potential disease gene & {$[31]$} \\
\hline NBN & Potential disease gene & {$[32]$} \\
\hline PARP1 & Potential biomarker & {$[33,34]$} \\
\hline NCOR2 & Potential biomarker & [35] \\
\hline RXRA & Potential therapeutic target & {$[36]$} \\
\hline WRN & Potential disease gene & [37] \\
\hline EXO1 & Potential disease gene & [38] \\
\hline NCOA3 & Potential disease gene & [39] \\
\hline RMI2 & Potential disease gene & {$[40]$} \\
\hline TOPBP1 & Potential therapeutic target & {$[41]$} \\
\hline \multicolumn{3}{|c|}{$x^{2}$} \\
\hline HRAS & Potential disease gene & {$[42]$} \\
\hline \multicolumn{3}{|l|}{ HAUS7 } \\
\hline \multicolumn{3}{|l|}{ CEP72 } \\
\hline GTF2I & Potential disease gene & {$[43]$} \\
\hline BCLAF1 & Potential disease gene & {$[44]$} \\
\hline \multicolumn{3}{|l|}{ HAUS3 } \\
\hline FGFR1OP & Potential disease gene & {$[45,46]$} \\
\hline \multicolumn{3}{|l|}{ CEP131 } \\
\hline \multicolumn{3}{|l|}{ GPR83 } \\
\hline ALMS1 & Potential disease gene & {$[47]$} \\
\hline \multicolumn{3}{|l|}{ AD } \\
\hline MAP2 & Potential disease gene & {$[48]$} \\
\hline \multicolumn{3}{|l|}{ DPYSL3 } \\
\hline ERRFI1 & Potential disease gene & [49] \\
\hline DAB2 & Potential disease gene & {$[50]$} \\
\hline AMPH & Potential disease gene & [51] \\
\hline SYN1 & Potential disease gene & {$[52]$} \\
\hline SYT9 & Potential disease gene & [53] \\
\hline \multicolumn{3}{|l|}{ AXIN1 } \\
\hline PRNP & Potential disease gene & {$[54]$} \\
\hline AAK1 & Potential disease gene & {$[55]$} \\
\hline
\end{tabular}

specific, static PPI networks downloaded from online databases contain many false positives, and directly using them would limit the accuracy of disease gene prediction. Moreover, for patients with a specific disease, their disease states might be driven by different subset of disease genes, and analyzing their data together might affect the identification of rarely mutated disease genes .

Therefore, in this study, an ensemble algorithm is proposed to predict disease genes from clinical sample-based networks. The algorithm first constructs single samplebased networks by combining clinical samples and a universal static PPI network. A group of networks which contain disease-related PPIs are generated. Then, case samples are divided into different clusters and networks belong to the samples in the same cluster are merged together. This step allows patients with similar causing genes to be analyzed together. After that, 0-1 centrality features extracted from the fused networks are used to train the logistic models that calculate the probability of each genes being disease-associated in each fused network. Finally, an ensemble strategy is performed by choosing the maximum probability obtained from different fused networks as the final probability of a gene being disease-associated.

In the experiments conducted on $\mathrm{BC}, \mathrm{TC}$ and $\mathrm{AD}$, our EdgCSN is much better than the competing algorithms in terms of AUC scores. Further analysis of the top 10 unknown genes also illustrate that EdgCSN is capable of predicting novel disease genes. Our study has provided insight into how clustering patient samples might improve the prediction of disease genes.

\section{Conclusions}

Our EdgCSN use ensemble learning to predict disease genes from clustered sample-based networks. In the future, the strategies used for clustering can be further improved. For instance, Eq. (2) uses the expression data of all the genes to calculate the pairwise distances, and the results might be dominated by non-disease genes. We could reduce the number of genes used for clustering and choose those differentially expressed genes or marker genes that are associated with a specific subtype. These subsets of genes should improve the clustering results as well as the final prediction.

\section{Abbreviations}

AD: Alzheimer's disease; AUC: Area under the curve; BC: Breast cancer; DGN: Disease gene network; EdgCSN: Ensemble disease gene prediction by $\mathbf{C l i n i c a l}$ Sample-based Networks; FN: False negative; FP: False positive; FPR: False positive rate; LOOCV: Leave one out cross validation; PPI: Protein-protein interaction; ROC: Receiver operating characteristic; TC: Thyroid cancer; TN: True negative; TP: True positive; TPR: True positive rate

\section{Acknowledgements}

This work is supported in part by Natural Science and Engineering Research Council of Canada (NSERC), China Scholarship Council (CSC) and by the National Natural Science Foundation of China under Grant No. 61772552 and No. 61602386, and the Natural Science Foundation of Shaanxi Province under Grant No. 2017JQ6008.

\section{About this supplement}

This article has been published as part of BMC Bioinformatics Volume 21 Supplement 2, 2020: Selected articles from the 6th International Work-Conference on Bioinformatics and Biomedical Engineering. The full contents of the supplement are available online at URL.

\section{Authors' contributions}

$\mathrm{PL}$ conducted the bioinformatics analysis, and FXW supervised the study. PL and FXW wrote the manuscript. All authors read, revised and approved the final version of the manuscript.

\section{Funding}

The publication costs are funded by Natural Science and Engineering Research Council of Canada (NSERC).

\section{Availability of data and materials}

The datasets generated and analyzed during the current study are available at: https://github.com/luoping1004/EdgCSN.

\section{Ethics approval and consent to participate}

Not applicable.

Consent for publication

Not applicable. 


\section{Competing interests}

The authors declare that they have no competing interests.

\section{Author details}

${ }^{1}$ Division of Biomedical Engineering, University of Saskatchewan, S7N 5A9 Saskatoon, Canada. ${ }^{2}$ School of Information, Beijing Wuzi University, 101149 Beijing, China. ${ }^{3}$ School of Computer Science, Northwestern Polytechnical University, 710072 Xi'an, China. ${ }^{4}$ School of Mathematics and Physics, University of South China, 421001 HengYang, China. ${ }^{5}$ Department of Computer Science, University of Saskatchewan, S7N 5C9 Saskatoon, Canada. ${ }^{6}$ School of Mathematics and Statistics, Hainan Normal University, 571158 Haikou, China.

${ }^{7}$ Department of Mechanical Engineering, University of Saskatchewan, S7N 5 A9 Saskatoon, Canada.

\section{Published: 13 March 2020}

\section{References}

1. Moody SE, Boehm JS, Barbie DA, Hahn WC. Functional genomics and cancer drug target discovery. Curr Opin Mol Ther. 2010;12(3):284-93.

2. Köhler $S$, Bauer $S$, Horn D, Robinson PN. Walking the interactome for prioritization of candidate disease genes. Am J Hum Genet. 2008;82(4): 949-58.

3. Gillis J, Pavlidis P. The impact of multifunctional genes on" guilt by association" analysis. PloS ONE. 2011;6(2):17258.

4. Chen B, Fan W, Liu J, Wu F-X. Identifying protein complexes and functional modules-from static ppi networks to dynamic ppi networks. Brief Bioinforma. 2013;15(2):177-94.

5. Jia P, Zheng S, Long J, Zheng W, Zhao Z. dmgwas: dense module searching for genome-wide association studies in protein-protein interaction networks. Bioinformatics. 2010;27(1):95-102.

6. Wang Q, Yu H, Zhao Z, Jia P. Ew_dmgwas: edge-weighted dense module search for genome-wide association studies and gene expression profiles. Bioinformatics. 2015150. https://doi.org/10.1093/bioinformatics/btv150.

7. Winter C, Kristiansen G, Kersting S, Roy J, Aust D, Knösel T, Rümmele P, Jahnke B, Hentrich V, Rückert F, et al. Google goes cancer: improving outcome prediction for cancer patients by network-based ranking of marker genes. PLoS Comput Biol. 2012;8(5):1002511.

8. Luo P, Tian L-P, Ruan J, Wu F-X. Identifying disease genes from ppi networks weighted by gene expression under different conditions. In: Bioinformatics and Biomedicine (BIBM), 2016 IEEE International Conference On. IEEE; 2016. p. 1259-1264. https://doi.org/10.1109/bibm. 2016.7822699.

9. Chen B, Shang X, Li M, Wang J, WU F-X. A two-step logistic regression algorithm for identifying individual-cancer-related genes. In: Bioinformatics and Biomedicine (BIBM), 2015 IEEE International Conference On. IEEE; 2015. p. 195-200. https://doi.org/10.1109/bibm. 2015.7359680.

10. Chen B, Shang X, Li M, Wang J, Wu F-X. Identifying individual-cancer-related genes by rebalancing the training samples. IEEE Trans Nanobiosci. 2016;15(4):309-15.

11. Singh-Blom UM, Natarajan N, Tewari A, Woods JO, Dhillon IS, Marcotte EM. Prediction and validation of gene-disease associations using methods inspired by social network analyses. PloS ONE. 2013;8(5):58977.

12. Luo $P$, Tian L-P, Chen B, Xiao Q, Wu F-X. Predicting disease genes from clinical single sample-based ppi networks. In: International Conference on Bioinformatics and Biomedical Engineering. Springer; 2018. p. 247-58. https://doi.org/10.1007/978-3-319-78723-7_21.

13. Wang B, Mezlini AM, Demir F, Fiume M, Tu Z, Brudno M, Haibe-Kains B, Goldenberg A. Similarity network fusion for aggregating data types on a genomic scale. Nat Methods. 2014;11(3):333.

14. Chen B, Wang J, Li M, Wu F-X. Identifying disease genes by integrating multiple data sources. BMC Med Genomics. 2014;7(Suppl 2):2.

15. Chen B, Li M, Wang J, Wu F-X. Disease gene identification by using graph kernels and markov random fields. Sci China Life Sci. 2014;57(11):1054-63.

16. Chen B, Li M, Wang J, Shang X, Wu F-X. A fast and high performance multiple data integration algorithm for identifying human disease genes. BMC Med Genomics. 2015;8(Suppl 3):2.

17. Katz L. A new status index derived from sociometric analysis. Psychometrika. 1953;18(1):39-43.

18. Lu Z, Savas B, Tang W, Dhillon IS. Supervised link prediction using multiple sources. In: 2010 IEEE 10th International Conference on Data
Mining (ICDM). IEEE; 2010. p. 923-8. https://doi.org/10.1109/icdm.2010. 112.

19. Luo P, Tian L-P, Ruan J, Wu F-X. Disease gene prediction by integrating ppi networks, clinical rna-seq data and omim data. IEEE/ACM Trans Comput Biol Bioinforma. 2019;16(1):222-32.

20. Hamosh A, Scott AF, Amberger J, Bocchini C, Valle D, McKusick VA. Online mendelian inheritance in man (omim), a knowledgebase of human genes and genetic disorders. Nucleic Acids Res. 2002;30(1):52-5.

21. Forbes SA, Beare D, Boutselakis H, Bamford S, Bindal N, Tate J, Cole CG, Ward S, Dawson E, Ponting L, et al. Cosmic: somatic cancer genetics at high-resolution. Nucleic Acids Res. 2016;45(D1):777-83.

22. Grossman RL, Heath AP, Ferretti V, Varmus HE, Lowy DR, Kibbe WA, Staudt LM. Toward a shared vision for cancer genomic data. N Engl J Med. 2016;375(12):1109-12.

23. Pachter L. Models for transcript quantification from rna-seq. arXiv preprint. 2011;1104(3889):.

24. Scheckel C, Drapeau E, Frias MA, Park CY, Fak J, Zucker-Scharff I, Kou Y, Haroutunian V, Ma'ayan A, Buxbaum JD, et al. Regulatory consequences of neuronal elav-like protein binding to coding and non-coding rnas in human brain. eLife. 2016;5:10421.

25. LiT, Wernersson R, Hansen RB, Horn H, Mercer J, Slodkowicz G, Workman CT, Rigina O, Rapacki K, Stærfeldt HH, et al. A scored human protein-protein interaction network to catalyze genomic interpretation. Nat Methods. 2016;14(1):61-4.

26. Love MI, Huber W, Anders S. Moderated estimation of fold change and dispersion for rna-seq data with deseq2. Genome Biol. 2014;15(12):550.

27. Tang $X$, Hu X, Yang $X$, Sun $Y$. A algorithm for identifying disease genes by incorporating the subcellular localization information into the protein-protein interaction networks. In: Bioinformatics and Biomedicine (BIBM), 2016 IEEE International Conference On. IEEE; 2016. p. 308-311. https://doi.org/10.1109/bibm.2016.7822537.

28. Chen Y, Wang W, Zhou Y, Shields R, Chanda SK, Elston RC, Li J. In silico gene prioritization by integrating multiple data sources. PloS ONE. 2011;6(6):21137.

29. Chen J, Bardes EE, Aronow BJ, Jegga AG. Toppgene suite for gene list enrichment analysis and candidate gene prioritization. Nucleic Acids Res. 2009:37(suppl_2):305-11.

30. O'hayre M, Vázquez-Prado J, Kufareva I, Stawiski EW, Handel TM, Seshagiri S, Gutkind JS. The emerging mutational landscape of g proteins and g-protein-coupled receptors in cancer. Nat Rev Cancer. 2013;13(6): 412.

31. Krøigård AB, Larsen MJ, Brasch-Andersen C, Lænkholm A-V, Knoop AS, Jensen JD, Bak M, Mollenhauer J, Thomassen M, Kruse TA. Genomic analyses of breast cancer progression reveal distinct routes of metastasis emergence. Sci Rep. 2017;7:43813.

32. Uzunoglu H, Korak T, Ergul E, Uren N, Sazci A, Utkan NZ, Kargi E, Triyak Ç, Yirmibesoglu O. Association of the nibrin gene (nbn) variants with breast cancer. Biomed Rep. 2016;4(3):369-73.

33. Ossovskaya V, Koo IC, Kaldjian EP, Alvares C, Sherman BM. Upregulation of poly (adp-ribose) polymerase-1 (parp1) in triple-negative breast cancer and other primary human tumor types. Genes Cancer. 2010;1(8):812-21.

34. Mazzotta A, Partipilo G, De Summa S, Giotta F, Simone G, Mangia A. Nuclear parp1 expression and its prognostic significance in breast cancer patients. Tumor Biol. 2016;37(5):6143-53.

35. Zhang L, Gong C, Lau SL, Yang N, Wong OG, Cheung AN, Tsang JW, Chan KY, Khoo U-S. Splicearray profiling of breast cancer reveals a novel variant of ncor2/smrt that is associated with tamoxifen resistance and control of er $\alpha$ transcriptional activity. Cancer Res. 2012;73(1):. https://doi. org/10.1158/0008-5472.can-12-2241.

36. Crowe DL, Chandraratna RA. A retinoid $x$ receptor (rxr)-selective retinoid reveals that $r \times r-\alpha$ is potentially a therapeutic target in breast cancer cell lines, and that it potentiates antiproliferative and apoptotic responses to peroxisome proliferator-activated receptor ligands. Breast Cancer Res. 2004;6(5):546.

37. Shamanna RA, Lu H, Croteau DL, Arora A, Agarwal D, Ball G, Aleskandarany MA, Ellis IO, Pommier Y, Madhusudan S, et al. Camptothecin targets wrn protein: mechanism and relevance in clinical breast cancer. Oncotarget. 2016;7(12):13269.

38. Zhang M, Zhao D, Yan C, Zhang L, Liang C. Associations between nine polymorphisms in exo1 and cancer susceptibility: a systematic review and meta-analysis of 39 case-control studies. Sci Rep. 2016;6:29270. 
39. Gupta A, Hossain MM, Miller N, Kerin M, Callagy G, Gupta S. Ncoa3 coactivator is a transcriptional target of $\mathrm{xbp} 1$ and regulates perk-eif2 $\alpha$-atf4 signalling in breast cancer. Oncogene. 2016;35(45):5860.

40. Yamamoto Y, Tsuchida A, Ushiwaka T, Nagai R, Matsumoto M, Komatsu J, Kinoshita H, Minami S, Hayashi K. Comparison of 4 risk-of-malignancy indexes in the preoperative evaluation of patients with pelvic masses: a prospective study. Clin Ovarian Other Gynecol Cancer. 2014;7(1-2):8-12.

41. Chowdhury P, Lin GE, Liu K, Song Y, Lin F-T, Lin W-C. Targeting topbp1 at a convergent point of multiple oncogenic pathways for cancer therapy. Nat Commun. 2014;5:5476.

42. Xing M. Clinical utility of ras mutations in thyroid cancer: a blurred picture now emerging clearer. BMC Med. 2016;14(1):12.

43. Petrini I, Meltzer PS, Kim I-K, Lucchi M, Park K-S, Fontanini G, Gao J, Zucali PA, Calabrese F, Favaretto A, et al. A specific missense mutation in gtf2i occurs at high frequency in thymic epithelial tumors. Nat Genet. 2014;46(8):844.

44. Galdiero F, Bello AM, Spina A, Capiluongo A, Liuu S, De Marco M, Rosati A, Capunzo M, Napolitano M, Vuttariello E, et al. Identification of bag3 target proteins in anaplastic thyroid cancer cells by proteomic analysis. Oncotarget. 2018;9(8):8016.

45. Santoro M, Carlomagno F. Central role of ret in thyroid cancer. Cold Spring Harb Perspect Biol. 2013;5(12):009233.

46. Bossi D, Carlomagno F, Pallavicini I, Pruneri G, Trubia M, Raviele PR, Marinelli A, Anaganti S, Cox MC, Viale G, et al. Functional characterization of a novel fgfr 1 op-ret rearrangement in hematopoietic malignancies. Mol Oncol. 2014;8(2):221-31.

47. Papadakis M, Meyer A, Schuster F, Weyerbrock N, Corinth C, Dotzenrath C. Follicular variant of papillary thyroid cancer in alström syndrome. Familial Cancer. 2015;14(4):599-602.

48. Xie C, Miyasaka T. The role of the carboxyl-terminal sequence of tau and map2 in the pathogenesis of dementia. Front Mol Neurosci. 2016;9:158.

49. Russo A. Decreased mitogen inducible gene 6 (mig-6) associated with symptom severity in children with autism. Biomark Insights. 2014;9:15218.

50. Song L, Gu Y, Jie J, Bai X, Yang Y, Liu C, Liu Q. Dab2 attenuates brain injury in app/ps1 mice via targeting transforming growth factor-beta/smad signaling. Neural Regen Res. 2014;9(1):41.

51. De Jesús-Cortés HJ, Nogueras-Ortiz CJ, Gearing M, Arnold SE, Vega IE. Amphiphysin-1 protein level changes associated with tau-mediated neurodegeneration. Neuroreport. 2012;23(16):942.

52. Neuner SM, Wilmott LA, Hoffmann BR, Mozhui K, Kaczorowski CC. Hippocampal proteomics defines pathways associated with memory decline and resilience in normal aging and alzheimer's disease mouse models. Behav Brain Res. 2017;322:288-98.

53. Gautam V, D'Avanzo C, Berezovska O, Tanzi RE, Kovacs DM. Synaptotagmins interact with app and promote $\mathrm{a} \beta$ generation. Mol Neurodegener. 2015;10(1):31.

54. Zhang W, Jiao B, Xiao T, Pan C, Liu X, Zhou L, Tang B, Shen L. Mutational analysis of prnp in alzheimer's disease and frontotemporal dementia in china. Sci Rep. 2016;6:38435.

55. Fu X, Ke M, Yu W, Wang X, Xiao Q, Gu M, Lü Y. Periodic variation of aak1 in an $\mathrm{a} \beta$ 1-42-induced mouse model of alzheimer's disease. J Mol Neurosci. 2018;65:179-89.

\section{Publisher's Note}

Springer Nature remains neutral with regard to jurisdictional claims in published maps and institutional affiliations.

\section{Ready to submit your research? Choose BMC and benefit from:}

- fast, convenient online submission

- thorough peer review by experienced researchers in your field

- rapid publication on acceptance

- support for research data, including large and complex data types

- gold Open Access which fosters wider collaboration and increased citations

- maximum visibility for your research: over 100M website views per year

At $\mathrm{BMC}$, research is always in progress.

Learn more biomedcentral.com/submissions 